\title{
Visión y propuestas con perspectiva de género para el progreso del Olimpismo en Colombia
}

\section{Gender perspective proposals for Colombian National Olympic Movement progress}

\author{
Catalina Melendro-Blanco' (iD
}

1Carterbury Christ Church University, Sport and Exercise Science Faculty. Canterbury, Kent, Inglaterra. Consultora en gobernanza deportiva, Académica de Número Academia Olímpica Colombiana. Bogotá D.C, Colombia catalina.melendro@uexternado.edu.co

Cómo citar: Melendro-Blanco, C. 2022. Visión y propuestas con perspectiva de género para el progreso del olimpismo en Colombia. Rev. Digit. Act. Fis. Deport. 8(1):e2154. http://doi.org/10.31910/rdafd.v8.n1.2022.2154

Artículo de acceso abierto publicado por Revista Digital: Actividad Física y Deporte, bajo una licencia Creative Commons CC BY-NC 4.0

Publicación oficial de la Universidad de Ciencias Aplicadas y Ambientales U.D.C.A, Institución de Educación Superior Acreditada de Alta Calidad por el Ministerio de Educación Nacional.

Recibido: agosto 15 de 2021 Aceptado: noviembre 4 de 2021 Editado por: Álvaro José Gracia Díaz

\section{RESUMEN}

Introducción: La historia de avances por la igualdad de género al interior del Comité Olímpico Internacional (COI) es y ha sido una historia de luchas de mujeres, quienes han soñado con participar en igualdad de condiciones y de oportunidades de la práctica deportiva; no obstante, la comprensión global de la igualdad de género, en el ámbito deportivo, se ha centrado en la igualdad numérica de atletas mujeres y hombres; por ello, existe la necesidad de contemplar y entender la transversalización de la perspectiva de género, desde un análisis holístico del sistema Olímpico Internacional. Objetivo: En este sentido, resulta perentorio analizar cuál es la realidad deportiva nacional colombiana hacia la igualdad de género, en el Movimiento Olímpico. Metodología: Para ello, se analizan los indicadores y los principios de buena gobernanza deportiva de la herramienta de evaluación del Observador Nacional de Gobernanza Deportiva, específicamente, asociados a la promoción de la equidad de género. Resultados: Al analizar los diversos resultados, se observa un patrón nacional de vacíos frente a la implementación de medidas direccionadas en la defensa de la equidad de género, debido al supuesto histórico que asume la promoción y la democratización del deporte, como un objetivo neutro al género, lo cual, ha conllevado a grandes brechas en detrimento de las mujeres y las niñas, en el Movimiento Olímpico nacional. Conclusiones: Se concluye, la responsabilidad del sistema olímpico nacional de diseñar, implementar, monitorear y evaluar políticas, estrategias, programas y acciones, con perspectiva de género para transformar las barreras adicionales, a las cuales, se enfrentan las mujeres y las niñas, para su aproximación y experiencia de la filosofía del olimpismo.

Palabras clave: Perspectiva de género; Olimpismo; Gobernanza deportiva; ComitéOlímpico Internacional.

\section{ABSTRACT}

Introduction: Historically, such progress as there has been in gender equality within the Olympic Movement is the result of women's advocacy for equality of opportunity and conditions. However, Sports Organizations' understanding of equality in this context has often focused only on numerical equalization of male and female competitors. Due 
to this, international studies related to gender equality within the Olympic movement highlight the need to implement gendermaintreaming processes from a holistic understanding of the International Olympic system and governance structure. Principal Objective: In this sense, it is imperative to examine progress in gender equality at the Colombian National Olympic Movement. Methodology: For this, sport good governance indicators and principles specifically associated with the promotion of gender equality from the National Sports Governance Observer for the Colombia country report, were analyzed. Results: The various results, showed a national pattern of "gaps \& lacks" without any positive result in the implementation of measures aimed at promoting gender equity within the Colombian national sport context due to the historical and cultural assumption that sport promotion and democratization is a genderneutral objective, which has been translated into large women and girls 'rights "gaps \& lacks" inside the Colombian Olympic Movement. Conclusion: There is a need for the Colombian National Olympic institutions to implement a gender mainstreaming strategy in the design, implementation, monitoring and evaluation of every policy, strategy, program or action in order to remove the additional barriers women and girls have historically faced to access and experience Olympism philosophy of life.

Keywords: Gender mainstreaming; Olympism; Sport governance; International Olympic Committee.

\section{INTRODUCCIÓN}

\section{Historia de avances por la igualdad de género al interior del Comité Olímpico Internacional.} Múltiples escritos y discursos se han elaborado para abordar el papel de la mujer en los juegos olímpicos (Coakley, 2009; Teetzel, 2011; Mitchell, 2012; Coche \& Tuggle, 2016). En su origen moderno, bajo la inspiración y liderazgo del Barón Pierre de Coubertin, el rol de las mujeres estaba asociado, únicamente, a la entrega de los premios, concepción asociada y comprendida por la época histórica en la que se inscribe el resurgimiento de los Juegos Olímpicos (JJ. OO.) y el nacimiento filosófico del olimpismo.

En efecto, el pensamiento de Coubertin frente a la presencia de mujeres en un estadio, como antiestética, poco interesante e incorrecta, no era ninguna particularidad de la época sino, por el contrario, una posición y creencia compartida por la gran mayoría de esa sociedad, en donde la igualdad o diversidad de género, eran temas inexistentes o completamente ignorados (Avena, 2017); sin embargo, 125 años han pasado y el Movimiento Olímpico ha venido mostrando, en cada una de las ediciones de los Juegos Olímpicos acaecidos en nuestra época moderna, avances en el número total y porcentual de mujeres participantes (Nunes, 2019).

El recuento histórico de mujeres participantes como deportistas en los Juegos Olímpicos modernos comenzó sin ninguna mujer presente en la primera edición, en Atenas 1996. Casi cincuenta años después, en Helsinki 1952, se logró llegar a una representatividad de $10 \%$ de mujeres deportistas, en las diversas justas deportivas. El $20 \%$, se consiguió hasta Montreal 1976 y el 40 \%, en la primera edición del siglo XXI, en Atenas 2004. En la actualidad, el Comité Olímpico Internacional (COI) exalta que, en Tokio 2021, se llegó a un nuevo récord del 48,8 \% de participación de mujeres y se tiene, de la misma manera, el compromiso de alcanzar la total igualdad de género para los Juegos Olímpicos de París 2024 (COI, 2020a).

Este avance inicial en el número de mujeres no surgió por la inercia del tiempo, sino por el contrario, se dio gracias a las voces, las demandas y las luchas de diversas mujeres que soñaban competir y participar en igualdad de condiciones de la práctica deportiva; en particular, Alice Milliat, nadadora y remadora francesa, tuvo un rol importantísimo desde el activismo feminista deportivo en la defensa de los derechos de las mujeres, para ser partícipes de la práctica atlética (Gicquel, 2021). Mencionar el rol de Milliat resulta fundamental para recordar que, este proceso de avances de la igualdad de género en el Movimiento Olímpico, es y ha sido una historia de luchas y de demandas de mujeres, quienes han soñado con crear un estilo de vida, basado en el deporte, la cultura, la educación y la cooperación, elementos conceptuales, que se encuentran en el origen mismo del olimpismo.

En la actualidad, los avances más recientes, en cuanto a la igualdad de género en el Movimiento Olímpico, han sido la consecuencia esperada de la recomendación número 11 de la Agenda Olímpica 2020, titulada "Igualdad de género" (COI, 2014). Esta recomendación invoca que la igualdad de género será promovida por el COI, por medio de la inclusión de 
eventos conjuntos mixtos y del trabajo directo con federaciones internacionales, con el fin de alcanzar el $50 \%$ de participación femenina, en los JJ.OO.

Adicional a estas medidas en pro del equilibrio descriptivo porcentual de géneros instaurados por el COI, esta entidad, bajo el mandato de Thomas Bach, ha logrado, igualmente, progresos sustanciales en el fomento de las oportunidades de desarrollo para mujeres, a nivel dirigente. Asimismo, desde la Comisión Ejecutiva del COI, se impulsó el cambio en las pautas del protocolo olímpico, con el fin de permitir que una atleta femenina y un atleta masculino de cada Comité Olímpico Nacional (CON) Ileven, en conjunto, la bandera durante la ceremonia de apertura de los Juegos Olímpicos de verano; paralelo a estas medidas, el COI comandó la creación de un reporte de igualdad de género en el Movimiento Olímpico. Este reporte fue realizado por un grupo de trabajo sobre igualdad de género, presidido por Marisol Casado, presidenta de la Federación Internacional de Triatlón, una de las únicas dos federaciones internacionales de la Asociación de Federaciones Internacionales Olímpicas de Verano (ASOIF), presididas, en la actualidad, por una mujer (Riquelme, 2020).

El reporte incluyó 40 entrevistas con representantes del COI, de las federaciones deportivas internacionales y de los Comités Olímpicos Nacionales; también, participaron expertos en el ámbito de la igualdad de género, el liderazgo deportivo y la prensa, así como académicos, atletas y líderes del sector privado. El resultado del reporte son 25 recomendaciones, en materia de igualdad de género, agrupadas en cinco temáticas: Deporte, Representación e imagen, Financiación, Puestos directivos, Recursos humanos, supervisión y comunicación (COI, 2018).

\section{La realidad de la igualdad de género en el contexto} y la realidad deportiva nacional. Estos avances en pro de la igualdad de género en el Movimiento Olímpico, como lo es la igualdad en la representación descriptiva de mujeres deportistas en la ceremonia deportiva internacional más importante, a nivel mundial, como los son los Juegos Olímpicos de verano e invierno, resulta un avance hacia un movimiento más diverso e inclusivo; sin embargo, estos avances en la equidad de género en el Movimiento Olímpico, en el ámbito internacional, deben ser igualmente conceptualizados, analizados y cuestionados en la singularidad y particularidad nacional colombiana.

En ese sentido, surge el interés por conocer, al detalle, cuál es la realidad nacional del Movimiento Olímpico colombiano frente a las propuestas y los avances por la igualdad de género, que se están impulsando en la actualidad, internacionalmente.

\section{MATERIALES Y MÉTODOS}

Para realizar este análisis, se consideró bibliografía especializada, relacionada con la implementación de principios de equidad de género en el sector deportivo, específicamente, en los resultados de la herramienta de evaluación de la buena gobernanza deportiva de la entidad danesa Play the Game, dentro del proyecto "Observador Nacional de Buena Gobernanza Deportiva" (National Sport Governance Observer, NSGO). En detalle, se estudiaron los resultados de los indicadores y los principios de buena gobernanza, afines con la defensa y la promoción de la equidad de género en las federaciones deportivas nacionales colombianas, analizadas en el reporte de caso, así como en el Comité Olímpico Colombiano (COC).

El instrumento de evaluación NSGO fue creado para medir y mejorar la gobernanza en las organizaciones deportivas, a través del establecimiento de redes nacionales e internacionales, el intercambio de buenas prácticas y el monitoreo de 46 principios de buena gobernanza deportiva, compuestos por 274 indicadores, los cuales, se encuentran divididos en 4 dimensiones: Transparencia, Procesos Democráticos, Control Interno y Responsabilidad Social (Geeraert, 2018) (Tabla 1). 
Tabla 1. Dimensiones y principios de buena gobernanza, establecidos por la herramienta de evaluación NSGO.

\begin{tabular}{|c|c|}
\hline $\begin{array}{c}\text { Dimensión } \\
\text { Buena Gobernanza NSGO }\end{array}$ & $\begin{array}{c}\text { Principio } \\
\text { Buena Gobernanza NSGO }\end{array}$ \\
\hline \multirow{7}{*}{ Transparencia } & 1. Publicación de documentos legales y políticas \\
\hline & 2. Asamblea General \\
\hline & 3. Decisiones de Junta Directiva \\
\hline & 4. Miembros de Junta Directiva \\
\hline & 5. Atletas y clubes \\
\hline & 6. Reporte Anual \\
\hline & 7. Remuneraciones \\
\hline \multirow{13}{*}{ Procesos Democráticos } & 8. Elecciones \\
\hline & 9. Balance en composición Junta Directiva \\
\hline & 10. Comité de Nominación \\
\hline & 11. Quórum \\
\hline & 12. Límites a los términos de cargos de elección \\
\hline & 13. Representación de los miembros \\
\hline & 14. Frecuencia reuniones Junta Directiva \\
\hline & 15. Participación de atletas \\
\hline & 16. Participación de jueces/zas \\
\hline & 17. Participación de entrenadores/as \\
\hline & 18. Participación de voluntarios/as \\
\hline & 19. Participación de empleados/as \\
\hline & 20. Política de Igualdad de Genero \\
\hline \multirow{14}{*}{ Control interno } & 21. Supervisión \\
\hline & 22. Procedimiento de renuncia \\
\hline & 23. Reglas de elegibilidad \\
\hline & 24. Estructura clara de gobierno \\
\hline & 25. Supervisión de gestión \\
\hline & 26. Comité de Auditoría \\
\hline & 27. Controles Financieros \\
\hline & 28. Auto Evaluación de Juntas \\
\hline & 29. Auditoría externa \\
\hline & 30. Código de Conducta \\
\hline & 31. Procesos de conflicto de intereses \\
\hline & 32. Procedimiento de queja \\
\hline & 33. Procedimiento de apelación \\
\hline & $\begin{array}{l}\text { 34. Calendario de reuniones de la Junta } \\
\text { Directiva }\end{array}$ \\
\hline \multirow{12}{*}{ Responsabilidad Social } & 35. Consultoría en Gobernanza Deportiva \\
\hline & 36. Mitigación de riesgos de salud \\
\hline & 37. Combatir el acoso sexual \\
\hline & 38. Antidopaje \\
\hline & 39. Inclusión social \\
\hline & 40. Anti Discriminación \\
\hline & 41. Equidad de Género \\
\hline & 42. Anti Amaño de Partidos \\
\hline & 43. Sostenibilidad Ambiental \\
\hline & 44. Carreras Duales \\
\hline & 45. Deporte para todos y todas \\
\hline & 46. Derechos de Atletas \\
\hline
\end{tabular}


El reporte de caso del NSGO para Colombia, se realizó con las federaciones nacionales de balonmano, fútbol, atletismo, tenis y natación, estipuladas como las organizaciones obligatorias del estudio comparativo mundial del NSGO (Arias Castaño et al. 2020).

En particular, los tres principios analizados relativos a la promoción de la equidad de género en el deporte, son el 20 "Política de igualdad de género", el 37 "Combatir el acoso sexual" y el 41 "Equidad de género", de la herramienta de evaluación de buena gobernanza NSGO, que están asociados a un total de 24 indicadores.

El primero de estos principios, se encuentra bajo la segunda dimensión de buena gobernanza, establecida por las herramientas de evaluación del NSGO, denominada "Procesos Democráticos". Bajo este principio de "Política de igualdad de género", la herramienta evalúa si las organizaciones promueven acciones afirmativas por medio de políticas de recursos humanos, que propendan por la contratación de mujeres en los procesos de selección y de elección interna. Igualmente, evalúa, en específico, el balance en la representación de las mujeres en los cargos de dirigencia deportiva y en las diversas comisiones y comités deportivos.

Por su parte, tanto el segundo como el tercer principio, se encuentran bajo la cuarta dimensión de buena gobernanza del NSGO, denominada "Responsabilidad social". El segundo principio aborda el análisis de procesos y documentos que están direccionados a combatir el acoso sexual, estimulando la conformación de instancias que promuevan, activamente, la defensa de la integridad sexual y psicológica de deportistas, quienes han sido históricamente vulnerados, debido a las jerarquías de poder establecidas en el sector deportivo, entre entrenadores, directivos y deportistas, en particular, mujeres y niñas. Este principio, en concreto, analiza la difusión de información especializada y de mejores prácticas destinadas a combatir el acoso y el abuso sexual, en la especificidad del sistema deportivo federado, así como la existencia de procesos, la reglamentación deportiva y la implementación de instancias destinadas a recibir e investigar las quejas y denuncias de acoso y abuso sexual.

El tercer principio, denominado "Equidad de género", analiza las estrategias, las campañas, las políticas o procesos adelantados, tanto internamente como por medio de cooperación con otras organizaciones, para la promoción de la equidad de género, en la práctica deportiva. En específico, estudia la existencia y la difusión de material educativo para los y las tomadores/as de decisiones acerca de la transversalización de la equidad de género en el deporte, así como la existencia de puntos de contacto de género y de diversidad (Geeraert, 2018).

La existencia de estos tres principios de buena gobernanza y sus indicadores asociados con la defensa de la equidad de género y los derechos de las mujeres y niñas, en el ámbito deportivo federado, está sustentada bajo la premisa de la relevancia de la equidad de género, como elemento primordial de justicia, de democracia y de inclusión social y, por lo tanto, se encuentra en la base misma de la legitimidad del sistema deportivo (Ordóñez, 2017). Adicionalmente, tal como lo subrayan los autores de la herramienta de evaluación de la buena gobernanza, estos indicadores y principios, se basan en el rol social de las federaciones deportivas, que se constituyen, desde su concepción misma, en organizaciones con la misionalidad de crear conciencia de la importancia de la práctica deportiva en toda la sociedad, así como de la promoción y la organización de competencias deportivas.

\section{RESULTADOS Y DISCUSIÓN}

Al analizar los datos obtenidos por la herramienta de evaluación del Observador Nacional de la Gobernanza, se advierte, a nivel general, que dentro de los principios, en donde los organismos deportivos colombiano estudiados obtuvieron los resultados porcentuales más bajos, se encuentran los tres principios directamente relacionados con la defensa, el reconocimiento y la transformación de las disparidades y brechas existentes entre hombres y mujeres, en todos los aspectos estratégicos y estructurales, de los deportes analizados.

En términos generales, en el reporte de la evaluación de gobernanza en Colombia, el resultado promedio, para el principio concerniente a la política de equidad de género, fue el más bajo, de los 46 principios estudiados, con un valor de $8 \%$; luego, el principio referente a la equidad de género y el de prevención de acoso sexual, tuvieron, de igual manera, resultados muy por debajo de la media, con promedio del 15 y $22 \%$, respectivamente, lo cual, los ubica en las categorías de "no cumplimiento" y "debilidad", de la herramienta de evaluación (Geeraert, 2018) (Tabla 2). 
Tabla 2. Puntajes de las Federaciones colombianas encuestadas en los principios 20, 37 y 41 del NSGO.

\begin{tabular}{|l|c|c|c|}
\hline Principio & $\begin{array}{c}\text { 20. Política de } \\
\text { igualdad de } \\
\text { género }\end{array}$ & $\begin{array}{c}\text { 37. Combatir el } \\
\text { acoso sexual }\end{array}$ & $\begin{array}{c}\text { 41. Equidad de } \\
\text { Género }\end{array}$ \\
\hline FECODATLE & $0 \%$ & $0 \%$ & $0 \%$ \\
\hline FCF & $17 \%$ & $75 \%$ & $17 \%$ \\
\hline FC Balonmano & $0 \%$ & $0 \%$ & $0 \%$ \\
\hline FECNA & $0 \%$ & $0 \%$ & $0 \%$ \\
\hline FCT & $17 \%$ & $17 \%$ & $17 \%$ \\
\hline COC & $33 \%$ & $83 \%$ & $67 \%$ \\
\hline FCC & $0 \%$ & $8 \%$ & $17 \%$ \\
\hline FCE & $0 \%$ & $0 \%$ & $0 \%$ \\
\hline Promedio & $8 \%$ & $22 \%$ & $14,75 \%$ \\
\hline
\end{tabular}

Fuente: Arias Castaño et al. (2020).

Estos resultados, ponen de manifiesto las enormes falencias y vacíos del sistema deportivo olímpico colombiano frente al compromiso de no discriminación e inclusión de mujeres y niñas, tanto en la práctica deportiva como en las diversas áreas organizacionales y de dirigencia deportiva, del sistema deportivo federado. De hecho, tal como se observa en los resultados de la tabla 1 , de los 24 resultados obtenidos por las siete organizaciones deportivas olímpicas analizadas, tan solo uno de ellos, se encuentra en el rango de cumplimiento, mientras que, en trece, el resultado es nulo, sin ninguna medida que ofrezca protección y promoción de los derechos de mujeres y niñas, en el sector deportivo.

Luego, al analizar los resultados por Federaciones nacionales, se observa, igualmente, que la Federación Colombiana de Atletismo (FEDECODATLE), la Federación Colombiana de Balonmano (FCBalonmano), la Federación Colombiana de Natación (FECNA), la Federación Colombiana de Tenis (FCT), la Federación Colombiana de Ciclismo (FCC) y la Federación Colombiana de Esgrima (FCE) tienen todos los indicadores relacionados con la equidad de género, en la categoría de no cumplimiento.

De esta forma, se detalla que los resultados nacionales en equidad de género en el Movimiento Olímpico nacional no se encuentran alineados con la realidad, los avances y los esfuerzos que se están impulsando, a nivel internacional, por parte del Comité Olímpico Internacional.
Se pone de manifiesto así, que existe un patrón nacional de vacíos frente a la implementación de medidas direccionadas en la defensa de la equidad de género, en las diversas federaciones deportivas analizadas. Resultados muy diferentes frente a los que el Movimiento Olímpico internacional promueve y defiende, en cuanto a la igualdad de género, en el ámbito internacional.

Esto reside, en términos generales, en la concepción misma de lo que es la igualdad de género en la práctica deportiva, tanto a nivel nacional, en Colombia como a nivel regional, en el contexto latinoamericano. En efecto, al analizar las actuaciones y los discursos de las diversas federaciones nacionales, se dilucida que, históricamente, han asumido la promoción y la democratización del deporte como un objetivo neutro al género, por lo cual, no contemplan creación y diseño de acciones afirmativas que entiendan y transformen las brechas de género históricas, que recaen sobre mujeres y niñas, para su desarrollo en la práctica deportiva y, por ende, en su aproximación y vivencia del olimpismo, como filosofía de vida.

De hecho, la constatación de esta situación, va en detrimento completo del segundo principio fundamental del Olimpismo, expuesto en la Carta Olímpica, que es poner siempre el deporte al servicio del desarrollo armónico del ser humano, con el fin de favorecer el establecimiento de una sociedad pacífica y comprometida con el mantenimiento de la dignidad humana (COI, 2020b). 
Por ello, se debe cuestionar si es posible lograr este segundo principio del olimpismo, anteriormente citado, de poner el deporte al servicio del desarrollo armónico del ser humano, sin contemplar, analizar y transformar las prácticas culturales e institucionales discriminatorias hacia mujeres y niñas, en su acceso, goce y disfrute de la práctica deportiva.

La respuesta a esta pregunta es clara y no presenta ambigüedades: asumir la práctica deportiva, como una actividad neutra al género, desconoce las barreras sociales, culturales, económicas y deportivas, que experimentan las mujeres y niñas, lo cual, conlleva a la perpetuación de brechas de género en la práctica deportiva, que persisten en el tiempo, limitando el derecho a una vida sana y dificultando el encuentro del olimpismo, como filosofía de vida, para mujeres y niñas.

Aunque las cifras en la élite del deporte ilustran un avance constante en la participación descriptiva de mujeres deportistas en los JJ.OO, gracias a las diversas medidas implementadas por el COI, en el ámbito internacional, a nivel de la práctica deportiva amateur, en todos los países, según el reporte de Naciones Unidas acerca de la intersección de género, raza y discriminación (ONU, 2020) y la Declaración de Brighton + Helsinki sobre Mujeres y Deportes (IWG, 2014), el porcentaje de participación de mujeres y niñas en el deporte y la actividad física es siempre menor que la de hombres y niños. Esto significa, por consiguiente, que el género es aún, en la actualidad, una variable que se constituye en una barrera para poder aproximarse al olimpismo, como filosofía de vida, por lo cual, se subraya la necesidad de implementar la perspectiva de género, como motor del olimpismo, en Colombia.

Para el caso nacional y en específico, en las cifras distritales de Bogotá, en la última Encuesta Bienal de Culturas (EBC) disponible, se obtuvo que $51 \%$ de hombres y niños practicaban algún deporte de forma habitual, mientras que, para las mujeres y niñas, el resultado de práctica, era de tan solo el $27 \%$. Luego, cuando se preguntaba por la participación en equipo de deporte competitivo, la brecha porcentual de género se mantiene, con tan solo $10 \%$ de mujeres y niñas, quienes afirman haber participado en torneos o campeonatos deportivos, mientras que el porcentaje en hombres es de $24 \%$, frente a esta misma pregunta (SCRD, 2017).
De la misma forma, el enunciado de estas cifras locales, se encuentra también en los resultados globales, obtenidos por ONU Mujeres, en el estudio publicado en 2020, titulado "La COVID-19, las Mujeres, las Niñas y el Deporte" (ONU Mujeres, 2020).

En este estudio, se pone de manifiesto que los impactos de la pandemia se encuentran afectando, de manera más severa, a las mujeres y a las niñas, en muchas áreas de su vida, dentro de ella y con mayor severidad, en el ámbito deportivo, como consecuencia de desigualdades de género, que no se han contemplado, plenamente, como variables a entender en las soluciones y en las alternativas de propuestas, por los entes deportivos.

Adicionalmente, en el estudio, se proyecta también que, "una vez que se suspendan las medidas de confinamiento, muchas niñas no podrán regresar a la práctica deportiva, ya que los papeles tradicionales asociados al género volverán a afianzarse y sus contribuciones al cuidado de la familia serán vistos como necesarios para el bienestar e incluso la sobrevivencia de las familias, haciendo que se considere y asuma como subsidiaria la práctica deportiva de mujeres y niñas"; de esta forma, reportes y proyecciones, como el de ONU Mujeres, prevén la disminución de tiempo destinado a la práctica deportiva por parte de mujeres y de niñas, debido a los roles de género, que recaen sobre ellas.

Como consecuencia de la falta de acciones afirmativas que reconozcan la construcción histórica, social y económica, que desvaloriza el ser mujer en la práctica deportiva, tiene como resultado que la variable de género femenino, se constituya en una de las variables con mayor incidencia negativa en la práctica deportiva $y$, por consiguiente, en el desarrollo del olimpismo en Colombia. Sin oportunidades reales y comprensivas de las barreras y las limitaciones de mujeres y de niñas, el número de las mismas, pudiendo aproximarse y vivenciar la filosofía del olimpismo, está limitada a la exclusividad del privilegio o la necesidad de la excelencia.

Por ello, se debe impulsar la necesidad de estudiar, comprender y transformar las barreras y los obstáculos que enfrentan mujeres y niñas, para poder ser parte de la vivencia misma del olimpismo. Resulta así, particularmente perentorio, diseñar, implementar, monitorear y evaluar políticas, 
estrategias, programas y acciones, con perspectiva de género, para el desarrollo del olimpismo en Colombia, sin discriminación hacia mujeres y niñas, en todas sus diferencias y diversidades.

\section{Aportes para la transversalización de la perspectiva} de género en la práctica deportiva. Un primer punto de partida es el análisis de los seis factores básicos necesarios para alcanzar la igualdad de género, propuestos por el COI, en su reporte publicado en 2018.

El primer factor expuesto es el relativo a los valores que debe tener y defender la dirigencia de las organizaciones deportivas, en cuestiones de igualdad de género. Este primer factor resalta la necesidad de crear un marco teórico concerniente a la cultura y a los valores organizacionales, como variables "blandas" de liderazgo y buena gobernanza deportiva. De hecho, la buena gobernanza deportiva debe ser entendida desde un sistema conceptual, amplio y específico, del sistema deportivo. Tal como lo expone Sport Wales, la buena gobernanza deportiva está compuesta, tanto de variables "blandas" como de variables "duras"; por un lado, estas últimas, se componen de los sistemas, las políticas, los procedimientos y los reglamentos, elementos absolutamente claves para cualquier organización. Por otro lado, la gobernanza blanda hace referencia a los comportamientos, los valores, la ética y la integridad de la dirigencia deportiva. Este análisis conceptual, se basa en la concepción misma del deporte, como actividad social, que gira entorno a las personas: deportistas, voluntarios/as, entrenadores/ as, jueces/zas, padres y madres, subrayando que la gente está en el mismísimo corazón del deporte, lo que significa, que su bienestar debe estar en el centro de todas las organizaciones deportivas (Sport Wales, 2020). Esta realidad, a su vez, infiere que la relevancia debe tener, a nivel nacional, en el Comité Olímpico Colombiano, al constituirse en el faro del conocimiento y la promoción, tanto de los principios éticos universales de no discriminación como de acciones de inclusión y diversidad, promovidas por el Movimiento Olímpico, a nivel internacional.

El segundo factor es el relativo a la distribución de fondos específicos para mujeres deportistas y el uso de la financiación, como incentivo para la adopción de medidas que promuevan la igualdad de género. Mediante este factor, el COI subraya la importancia de transversalizar la perspectiva de género en las finanzas mismas de cada organización, perteneciente al Movimiento Olímpico, diferenciando, efectivamente, los recursos que se están destinando, teniendo en cuenta, particularmente, a las mujeres, desde sus necesidades y experiencias particulares y desestimulando así la neutralidad financiera al género en las inversiones deportivas, con el fin de garantizar el acceso al goce efectivo de sus derechos, en el Movimiento Olímpico nacional.

El tercer factor expuesto hace referencia a la adopción de una cultura de inclusión en la organización. Este factor es característicamente central, pues subraya cómo las organizaciones deportivas no deben limitarse a la "no discriminación", sino que deben trabajar activamente por la inclusión de grupos sociales, como las mujeres y niñas, quienes han sido excluidas y discriminadas, históricamente, de la práctica deportiva.

El cuarto factor, se centra en la preocupación de que las mujeres tengan no solo la oportunidad sino también el apoyo institucional para ocupar puestos de alta dirección, con influencia en la toma de decisiones. Este factor pone de manifiesto cómo la representación descriptiva de mujeres en los órganos deportivos no basta, sino que debe venir acompañada por medidas que permitan ocupar posiciones de decisión, en donde se espera aporten propuestas de representación, esenciales para fortalecer la participación futura de más mujeres y niñas.

El quinto factor hace referencia a la necesidad de usar la reglamentación legal de las entidades deportivas, por medio de estatutos, normativas y procesos electorales, como medios institucionales, necesarios para lograr afianzar la igualdad de género, en la estructura de cada organización.

El sexto y último factor indicado en el reporte es el relativo al seguimiento del progreso, la medición y la evaluación de los resultados de la igualdad de género y el fomento de la rendición de cuentas, con perspectiva de género. Este factor cobra particular importancia en la lógica de mejoramiento continuo, permitiendo analizar, desde una perspectiva de género crítica, las posibles falencias en el diseño programático y proponer, sobre ello, mejoras que tengan en cuenta las causas directas e indirectas de las mismas. 


\section{CONCLUSIONES}

Teniendo en cuenta lo anteriormente expuesto, es necesario, en primera medida, reconocer que la práctica deportiva es una acción que se vivencia de forma diferenciada, de acuerdo al género de los y las participantes. Variables como: los medios económicos, las creencias socioculturales frente a la práctica deportiva, el tiempo disponible para la práctica deportiva y el apoyo familiar, se constituyen en factores diferenciadores para mujeres y niñas, en el momento de decidir si pueden o quieren participar en el deporte, en la actividad física y en la competencia, por lo cual, es pertinente, hoy, más que nunca, la necesidad de trabajar en ofrecer oportunidades efectivas de participación, a mujeres y niñas, que entiendan y transformen estas desigualdades $y$ discriminaciones, basadas en el género.

En segunda instancia, los resultados en los diferentes indicadores, relativos a la equidad de género en los mayores entes deportivos nacionales en Colombia, ponen de manifiesto, tanto un vacío programático en el diseño e implementación de estrategias alineadas con el principio de no discriminación, que se encuentra en el corazón mismo del Olimpismo, como un desconocimiento teórico del sistema nacional del deporte, en la comprensión holística de las causas y las especificidades de las inequidades de género, en la práctica deportiva.

En tercera medida y como consecuencia de las dos anteriores, resulta particularmente necesario, estudiar, analizar y comprender las buenas prácticas que se vienen desarrollando, a nivel internacional, bajo los lineamientos del COI, encaminadas a poner en marcha acciones afirmativas, que reconozcan la construcción histórica, social y económica, que desvaloriza el ser mujer en la práctica deportiva y, con base en ese conocimiento, se adapten acciones con perspectiva de género, en el marco del contexto deportivo y sociocultural nacional, que permitan, positivamente, transformar las barreras adicionales, a las cuales, se enfrentan mujeres y niñas, con el fin de permitir y promover su encuentro, descubrimiento y apropiación de la filosofía del olimpismo.

El Movimiento Olímpico tiene un lugar cumbre en el mundo del deporte, lo que conlleva una gran responsabilidad. El olimpismo puede ser el mayor motor de equidad de género en el presente y futuro de la humanidad, pero, para ello, es necesario transversalizar la perspectiva de género en toda la cosmovisión de la filosofía olímpica. Integrar plenamente una perspectiva de género en todas las áreas procesos y estructuras, introduciendo revisiones, acciones, actividades, códigos y políticas con perspectiva de género, le permitirá al Movimiento Olímpico colombiano, no solo desarrollar adaptación, resiliencia y posición de liderazgo, sino, primordialmente, cumplir con su misión social y educativa, en su máxima expresión y máxima inclusión, posicionándose como el mejor ejemplo de equidad de género, en el escenario nacional, regional y local.

\section{REFERENCIAS}

1. ARIAS CASTAÑO, A.M.; RAMOS ACOSTA, J.; GÓMEZ SOLANO, J.H.; ORDOÑEZ SAAVEDRA, N.; SANDINO RODRÍGUEZ, M.C.; CARDONA MEJÍA, L.M. 2020. Informe estudio en Colombia (fase1): "Gobernanza en el deporte: la legitimidad en las federaciones deportivas nacionales" Disponible desde Internet en: http://www.coc.org.co/wp-content/ uploads/INFORME-INVESTIGACION-U.D.C.A.PLAY-THE-GAME-FASE-1.-FEDERACONESDEPORTIVAS-2019-1.pdf (con acceso el 15/09/2021).

2. AVENA, A. 2017. Gender equality in the Olympic Movement: not a simple question, not a simple answer. Journal of the Philosophy of Sport. 44(3):329-341.

https://doi.org/10.1080/00948705.2017.1359 616

3. COAKLEY, J.J. 2009. Sports in society: Issues and controversies (2nd Canadian ed.). McGraw-Hill (New York). 672p.

4. COCHE, R.; TUGGLE, C.A. 2016. The women's Olympics?: A gender analysis of NBC's coverage of the London 2012 Summer Games. Electronic News. 10(2):121-138. https://doi.org/10.1177/1931243116647770

5. COMITÉ OLÍMPICO INTERNACIONAL, COI. 2014. Olympic Agenda 2020- 20+20 Recommendations. Disponible desde Internet en: https://stillmed.olympic.org/ Documents/Olympic_Agenda_20200lympic_ Agenda_2020-20-20 Recommendations-ENG.pdf 
6. COMITÉ OLÍMPICO INTERNACIONAL, COI. 2018. Examen sobre igualdad de género. Informe del COI sobre igualdad de género. Disponible desde Internet en: https://stillmedab.olympic.org/ media/Document\%20Library/OlympicOrg/ News/2018/03/IOC-Gender-Equality-ReportSPA.pdf (con acceso el 08/09/2021).

7. COMITÉ OLÍMPICO INTERNACIONAL, COI. 2020a. Factsheet women in the Olympic Movement. Disponible desde Internet en: https://stillmed.olympic.org/media/ Document\%20Library/Olympicorg / Factsheets-Reference-Documents/Womenin-the-Olympic-Movement/FactsheetWomen-in-the-Olympic-Movement.pdf

8. COMITÉ OLÍMPICO INTERNACIONAL, COI. 2020b. Carta olímpica. Comité International Olympique (Lausanne). Disponible desde Internet en: https://stillmed.olympic.org/ media/Document\%20Library/OlympicOrg/ General/ES-Olympic-Charter.pdf (con acceso el 10/09/2021).

9. GEERAERT, A. 2018. National Sports Governance Observer. Indicators and instructions for assessing good governance in national sports federations. Aarhus: Play the Game / Danish Institute for Sports Studies. 103p.

10. GICQUEL, T. 2021. Alice Milliat, une statue pour une pionnière du sport féminin mondial. France Sport. Disponible desde Internet en: https:// sport.francetvinfo.fr/omnisport/alice-milliatune-statue-pour-une-pionniere-du-sportfeminin-mondial (con acceso el 08/09/2021).

11. IWG. 2014. Brighton plus Helsinki2014 Declaration on Women and Sport. Disponible desde Internet en: https://iwgwomenandsport.org/ wp-content/uploads/2019/03/Brighton-plusHelsinki-2014-Declaration-on-Women-andSport.pdf

12. MITCHELL, S. 2012. Women's Participation in the Olympic Games 1900-1926. In: Cott, N. (Ed.), Volume 8/2 Professional and WhiteCollar Employments. K.G.Saur (Berlin, Boston). p.416-436.

https://doi.org/10.1515/9783110976380.416
13. NUNES, R. 2019. Women athletes in the Olympic Games. Journal of Human Sport and Exercise. 14(3):674-683.

https://doi.org/10.14198/jhse.2019.143.17

14. ONU MUJERES. 2020. La COVID-19, las Mujeres, las Niñas y el Deporte: Reconstruir Mejor. Disponible desde Internet en: https:// www.unwomen.org//media/headquarters/ attachments/sections/library/publications/2020/ brief-covid-19-women-girls-and-sport-es. pdf?la=es\&vs=2641 (con acceso el 08/09/2021).

15. ORDÓÑEZ, N. 2017. La legitimidad, como principio de la gobernanza. Revista Olímpica Digital. 54:89-91.

16. ORGANIZACIÓN DE LAS NACIONES UNIDAS, ONU. 2020. Intersection of race and gender discrimination in sport. Report of the United Nations High Commissioner for Human Rights. Disponible desde Internet en: $\quad$ https://scf21.sporthumanrights.org/ GetDigitalPrintPDF?pkMaterialID=85137 (con acceso el 18/09/2021).

17. RIQUELME, G. 2020. El deporte y la desigualdad: con Sorenstam habrá sólo dos presidentas olímpicas. Marca. Disponible desde Internet en: https://www.marca.com/golf/ opinion/2020/12/04/5fc9803a22601dea448b457f. html (con acceso el 08/09/2021).

18. SECRETARÍA DE CULTURA, RECREACIÓN Y DEPORTE, SCRD. 2017. Encuesta Bienal de Culturas. Observatorio de Culturas. Disponible desde Internet en: https://www. culturarecreacionydeporte.gov.co/es/culturaciudadana/subdireccion-observatoriode-culturas/encuesta-bienal-de-culturas/ encuesta-2017 (con acceso el 08/09/2021).

19. SPORT WALES. 2020. Governance and leadership framework for wales: building on success: by the sport sector, for the sport sector. Disponible desde Internet en: https://www.sport.wales/ files/0a26509a4b1cbd61460824c823653507. pdf (con acceso el 08/09/2021).

20. TEETZEL, S. 2011. Rules and reform: eligibility, gender differences, and the Olympic Games. Sport in Society. 14(3):386-398.

https://doi.org/10.1080/17430437.2011.557275 